\title{
Lineale Construction von Kegelschnitten aus theilweise imaginären Elementen.
}

\author{
Von F. Spath in Trient. \\ (In analytischer Behandlung.) \\ Einleitung.
}

Die Aufgabe, einen Kegelschnitt zu construieren, wenn fünf Punkte desselben gegeben sind, fasst nach der v on S t a u d' t'schen Theorie der imaginären Elemente drei besondere Fälle in sich: die Punkte können sämmtlich reell sein; es können drei reelle Punkte und ein Paar complex conjugierter, es können endlich ein reeller Punkt und zwei Paare complex conjugierter gegeben sein. Im ersten Falle gibt der Pa scal'sche Satz eine Lösung der Aufgabe; sind $A, B, C, D, E$ die fünf Punkte, ist $g$ eine beliebige Gerade durch $A$ und ist $X$ der zweite Curvenpunkt auf $g$, so liegen nach dem genannten Satze die Schnittpunkte $H, J, K$ je zweier Gegenseiten des einfachen Sechseckes $A X B C D E$ auf einer Geraden:

$$
\left.\left.\left.\begin{array}{ll}
A X \\
C D
\end{array}\right\} H \quad \begin{array}{ll}
X B \\
D E
\end{array}\right\} J \quad \begin{array}{l}
B C \\
E A
\end{array}\right\} K
$$

Es ist somit der Punkt $X$ als Sehnittpunkt der Geraden $g$ und $B J$ mit Hilfe des Lineals allein construiert. In den beiden anderen Fällen wird die Aufgabe von Prof. Dr. E. W e y r in seinen Elementen der projectivischen Geometrie (II. Heft, pag. $157 \mathrm{ff}$.) durch Zurückführung auf den ersten Fall gelöst, und zwar im zweiten Falle durch eine lineare, im dritten durch eine quadratische Construction. Bei dieser Lösung wird von der Definition der imaginären Elemente weiter kein Gebrauch gemacht. Es ist nun zweckmäloig, den Begriff der complexen Elemente in der Geometrie nicht nur zur einheitlichen Fassung mehrerer sonst verschieden lautender Sätze oder Aufgaben zu verwenden, sondern denselben bei der Lösung der Aufgabe wirklich zu verwerten. Man kann ja vermöge der bekannten Erweiterung der Begriffe: Verbindungslinie und Schnittpunkt, auf imaginäre 
Elemente die Pascal'sche Construction auch auf den zweiten und dritten der obgenannten Fälle anwenden; denn die Giltigkeit des P a sca l'schen Satzes in diesem erweiterten Sinne ergibt sich in der analytischen Geometrie durch genau dieselbe Schlussweise, wie die des gewöhnlichen Pa scal'schen Satzes.

Die mechanische Anwendung des genannten Satzes auf unsere zwei Aufgaben würde zu verwickelten Constructionen führen; man würde sofort auf die bekanntlich quadratischen Constructionen von Verbindungslinien imaginärer Punkte und Schnittpunkten imaginärer Geraden stoßen. Erwägt man nun, dass, solange der zweite Curvenpunkt einer durch den reellen Punkt $A$ gehenden reellen Geraden gesucht wird, die besagte Construction auf einen und nur einen Punkt $X$ führt, so wird die Vermuthung nahe liegen, dass die quadratischen Hilfsconstructionen vermieden werden können. Bei gehöriger Berücksichtigung der auftretenden. Beziehungen ergibt sich in der That eine lineale Construction des Punktes $X$. Diese Beziehungen sind aber, besonders in der zweiten Aufgabe, nicht so naheliegend; um sie zu übersehen, bedürfen wir des leitenden Fadens der analytischen Geometrie. Es rührt dieser Gedanke von Prof. Dr. Otto Stolz in Innsbruck her. Bereits im Jahre 1886 theilte $\mathrm{Stolz}$ in seinen Vorlesungen über neuere analytische Geometrie je eine Lösung der beiden Aufgaben mit (s. u.) unter Andeutung des Weges, auf dem man dazu gelangt, und forderte mich auf, mich an der Herstellung der Beweise zu versuchen. Das Ergebnis dieses Versuches übergebe ich nun, von meinem verehrten Lehrer dazu aufgefordert, der Öffentlichkeit.

Zuvor muss jedoch der von Stolz in seinen Vorlesungen gepflegten analytischen Behandlung der imaginären Elemente gedacht werden, die er im IV. Bande der mathematischen Annalen bekannt gegeben hat. Die geometrische Deutung der imaginären Elemente in der Ebene wird auf analytischem Wege durch den Gedanken vermittelt, dass die Bedeutung der homogenen Coordinaten eines Punktes oder einer Geraden bei der Multiplication mit einer beliebigen, also auch complexen Constanten ungeändert bleibt. Hat man für die homogenen Coordinaten eines Punktes $\varrho x_{1}=\alpha_{1}+\beta_{1} i, \varrho x_{2}=\alpha_{2}+\beta_{2} i, \varrho x_{3}=\alpha_{3}+\beta_{3} i$, so liegt es nahe, das reelle Punktepaar $A\left(\alpha_{1}\left|\alpha_{2}\right| \alpha_{3}\right), B\left(\beta_{1}\left|\beta_{2}\right| \beta_{3}\right)$, zum complexen Punkte in Beziehung zu setzen. Da nun:

$$
\varrho(\sigma+\tau i) x_{r}=\sigma \alpha_{r}-\tau \beta_{r}+i\left(\sigma \beta_{r}+\tau \alpha_{r}\right), \quad(r=1,2,3),
$$

so kann der complexe Punkt mit den Coordinaten $\varrho x_{r}=\alpha_{r}+\beta_{r} i$ dem Complexe von reellen Punktepaaren mit den Coordinaten $\sigma \alpha_{r}-\tau \beta_{r}$ und $\boldsymbol{\tau} \alpha_{r}+\sigma \beta_{r}(r=1,2,3)$ zugeordnet werden, wobei $\sigma, \tau$ alle reellen Werte durchlaufen. Diese Punktepaare sind aber die Paare einer auf der Geraden $A B$ gelegenen elliptischen Involution, deren Doppelpunkte die Coordinaten $\alpha_{r} \pm i \beta_{r}$ besitzen. 
Die Unterscheidung der beiden derselben Involution zugeordneten complex conjugierten Punkte erfolgt nach der v on Staud t'schen Theorie, und zwar wird der im Sinne des Punktes $M_{k}\left(\varrho x_{r}=\alpha_{r}+k \beta_{r}\right)$ bei wachsendem $k$ beschriebenen Involution der Punkt $\alpha_{r}+i \beta_{r}$, bei abnehmendem $k$ der Punkt $\alpha_{r}-i \beta_{r}$ zugeordnet. Dieselben Bemerkungen lassen sich an die Gleichung des Punktes und der Geraden anknüpfen; die folgenden Rechnungen stützen sich auf eben diesen Umstand.

\section{Aufgabe.}

Der Kegelschnitt ist durch drei reelle Punkte $B, C, D$ und einem Paar complex conjugierter $\mathfrak{A}, \mathfrak{A}^{*}$ bestimmt; es soll der zweite Curvenpunkt $X$ der reellen, durch $B$ gehenden Geraden $g$ construiert werden.

Im folgenden sollen reelle Punkte stets durch große lateinische, complexe durch große deutsche Buchstaben bezeichnet werden, und zwar complex conjugierte Punkte durch denselben Buchstaben mit und ohne Sternchen.

Wir wenden auf das Sechseck $\mathfrak{H} * C B X D$ den Pascalschen Satz an.

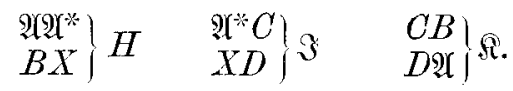

Die Punkte $H \Im$ Я liegen auf einer Geraden. Die lineale Construction der Geraden $D X$ lässt sich hier auch ohne Hilfe der analytischen Geometrie finden. Zunächst können wir aus dem Bestehen des Pascal'schen Satzes auf folgenden Satz schließen.

Wird der complexe Punkt $\Re$ mit $H$, einem Punkte des reellen Trügers von $\mathfrak{A}$, und der Punkt $\mathfrak{A}^{*}$ mit einem Punkte $C$ des reellen Trägers $(B C)$ von $\Re$ verbunden, so geht der reelle Träger $(X D)$ des Schnittpunktes $\&$ der beiden Verbindungs. linien durch den reellen Punkt $D$ der Verbindungslinie $\mathfrak{A} \Re$. (Dieser Satz fällt zusammen mit dem auf die Punkte $H, \mathfrak{A}, \mathfrak{I}^{*}$; $C, \Re^{*}, \mathfrak{\Omega}$ angewandten Satze von Pappus, nach welchem die Schnittpunkte:

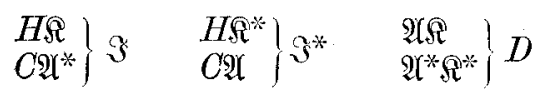

auf einer Geraden liegen.)

Um diesen Satz unmittelbar einzusehen, haben wir die Strahlen aufzusuchen, welche in den Involutionen $C \mathfrak{A}^{*}$, bezw. $H \Re$ dem gemeinsamen Strahle $C H$ conjugiert sind. Dem Punkte $C$ sei in der Involution $\Omega$ auf $B C$ der Punkt $C^{\prime}$ conjugiert. Schneidet $D C$ die Gerade $\mathfrak{H}^{*}$ in $E$, ist $E^{\prime}$ in der Involution $\mathfrak{A}$ dem Punkte $E, H^{\prime}$ dem Punkte $H$ zugeordnet, so ist $\mathfrak{H}^{*}$ 
definiert durch die Punktepaare $H H^{\prime}, E E^{\prime}$; stimmt außerdem der Sinn von $\mathfrak{A}^{*}$ mit dem durch die Punkte $H H^{\prime} E$ definierten Sinne überein, so ist der Sinn von $\mathfrak{A}$ durch die Aufeinanderfolge $E E^{\prime} H$ bestimmt. Die Schnittpunkte von $B C$ mit den Strahlen $D\left(E E^{\prime} H H^{\prime}\right)$ in dieser Aufeinanderfolge definieren den Punkt $\Re$. Ist $H_{1}$ der dritte, $H_{1}^{\prime}$ der vierte der genannten Schnittpunkte :

$$
\left.\left.\begin{array}{ll}
B C \\
D H
\end{array}\right\} H_{1}, \quad \begin{array}{ll}
B C \\
D H^{\prime}
\end{array}\right\} H_{1}^{\prime},
$$

so ist die Gerade $H \Im$ durch die Strahlen $H\left(C C^{\prime} H_{1} H_{1}^{\prime}\right)$ oder $H\left(C C^{\prime} D H^{\prime}\right)$, und zwar in dieser Folge definiert. Da die Doppelverhältnisse $C\left(H H^{\prime} E E^{\prime}\right)$ und $H\left(C C^{\prime} D H_{1}^{\prime}\right)$ gleich sind, so liegen auf einer Geraden die Schnittpunkte der Strahlenpaare

$$
\left.\left.\left.\begin{array}{ll}
C H^{\prime} \\
C^{\prime} I I
\end{array}\right\} \quad \begin{array}{ll}
C E \\
H D
\end{array}\right\} D \quad \begin{array}{l}
C E^{\prime} \\
H H_{1}^{\prime}
\end{array}\right\}
$$

Diese Gerade ist der reelle Träger des Schnittpunktes 3 der complexen Geraden $C \mathfrak{A}^{*}, H \Re$ und geht also durch die Punkte $D$ und J. Die Construction des Punktes $X$ ist also folgende: „Schneidet $B g$ den Träger der complexen Punkte $\mathfrak{A P}^{*}$ in $H, C D$ denselben in $E$, sind $H^{\prime}$, bezw. $E^{\prime}$ die in der Involution al conjugierten Punkte, so suche man die Schnittpunkte:

$$
\left.\left.\begin{array}{l}
D E^{\prime} \\
B C
\end{array}\right\} C^{\prime} \quad \begin{array}{ll}
H C^{\prime} \\
H^{\prime} C
\end{array}\right\} J
$$

Die Gerade $D J$ schneidet $g$ im gesuchten Punkte $X$." $\mathrm{Es}_{\mathrm{s}}$ ist dies die von Stolz angegebene Construction.

Es möge noch kurz erwähnt werden, dass diese Construction eine andere Deutung zulässt. Der Punkt $J$ beschreibt bei der Drehung des Strahles $g$ um $B$ einen Kegelschnitt, welcher die Punkte $C D \mathfrak{A} \mathfrak{U}^{*} C^{\prime}$ enthält. Schneidet eine Sehne durch den gemeinsamen Punkt $C$ der beiden Kegelschnitte dieselben in $B$, bezw. $C^{\prime}$, eine Sehne durch den gemeinschaftlichen Punkt $D$ dieselben in $X$, bezw. $J$, so liegt der Schnittpunkt $(H)$ der Geraden $B X$ und $C^{\prime} J$ auf der Verbindungslinie der beiden anderen gemeinschaftlichen Punkte $\mathfrak{A}, \mathfrak{A}^{*}$ der beiden Kegelschnitte.

Die einfachen Sechsecke $\mathfrak{A} \mathfrak{A}^{*} B X C D$ und $\mathfrak{A}^{*} X B C D$ liefern in ähnlicher Weise lineale Constructionen.

Wir gehen nun dazu über, die erste Construction auf analytischem Wege zu gewinnen. Im folgenden sollen kleine lateinische Buchstaben complexe Zahlen, griechische nur reelle Zahlen bedeuten. Der analytischen Darstellung legen wir ein 
trimetrisches Coordinatensystem $x_{1}, x_{2}, x_{3}$ zugrunde, dessen Fundamentalpunkte zur Vereinfachung der Gleichungen zweckentsprechend angenommen werden. Sehneidet $B C$ den reellen Träger des Punktes $\mathfrak{A}$ in $B_{3}$, ist $B_{2}$ zu $B_{3}$ conjugiert, so sei $B B_{2} B_{3}$ das Coordinatendreieck. Die analytische Darstellung der gegebenen Elemente ist dann die folgende:

$\mathfrak{A}: \quad x_{1}=0 \quad x_{3}+\mu i x_{2}=0$

$\mathfrak{H}^{*}: \quad x_{1}=0 \quad x_{3}-\mu i x_{2}=0$

$B: \quad x_{2}=0 \quad x_{3}=0$

$C: \quad x_{2}=0 \quad x_{3}+\alpha x_{1}=0$

$D: \quad \varrho x_{1}=\eta_{1}, \quad \varrho x_{2}=\eta_{2}, \quad \varrho x_{3}=\eta_{3}, \quad \eta_{1} \geq 0, \quad \eta_{2} \geq 0$

$B g: \quad x_{3}+\beta x_{2}=0$.

Es ist die analytische Darstellung der Punkte $H, \Im, \Re$ zu suchen:

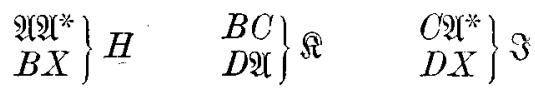

$\left.\left.\begin{array}{l}x_{1}=0 \\ x_{3}+\beta x_{2}=0\end{array}\right\} H \quad \begin{array}{l}x_{2}=0 \\ \left(\eta_{3}+\mu i \eta_{2}\right) x_{1}-\eta_{1}\left(x_{3}+\mu i x_{2}\right)=0\end{array}\right\} \hat{\Re}$

$\left.\begin{array}{rl}H \Re: \quad & \left(\eta_{3}+\mu i \eta_{2}\right) x_{1}-\eta_{1}\left(x_{3}+\beta x_{2}\right) \\ & \equiv\left[\left(\eta_{3}+\mu i \eta_{2}\right) x_{1}-\eta_{1}\left(x_{3}+\mu i x_{2}\right)\right]+\eta_{1}(\mu i-\beta) x_{2}=0 \\ C \mathfrak{A}^{*}: & \alpha x_{1}+x_{3}-\mu i x_{2}=0\end{array}\right\} \Im$

$D 9 X: \quad \eta_{1} \eta_{2}(\mu i-\beta)\left(\alpha x_{1}+x_{3}-\mu i x_{2}\right)-$

$-\left(\alpha \eta_{1}+\eta_{3}-\mu i \eta_{2}\right)\left[\left(\eta_{3}+\mu i \eta_{2}\right) x_{1}-\eta_{1}\left(x_{3}+\beta x_{2}\right)\right]=0$.

$D \mathcal{Y}$ ist wirklich eine reelle Gerade, denn der Coefficient von $\mu i$ in der Gleichung ist:

$$
\eta_{1} \eta_{2}\left[\left(\alpha x_{1}+x_{3}\right)+\beta x_{2}\right]-\alpha \eta_{1} \eta_{2} x_{1}-\eta_{1} \eta_{2}\left(x_{3}+\beta x_{2}\right) \equiv 0 .
$$

$D \Im X$ geht durch den Schnittpunkt der Geraden, deren Gleichungen durch die reellen Theile folgender Gleichungen gegeben sind :

$$
\begin{gathered}
(\mu i-\beta)\left(\alpha x_{1}+x_{3}-\mu i x_{2}\right)=0 \\
\left(\alpha \eta_{1}+\eta_{3}-\mu i \eta_{2}\right)\left[\left(\eta_{3}+\mu i \eta_{2}\right) x_{1}-\eta_{1}\left(x_{3}+\beta x_{2}\right)\right]=0 .
\end{gathered}
$$

Der reelle und der imaginäre Theil der ersten Gleichung stellen conjugierte Gerade der Involution $C \mathfrak{Q}^{*}$ dar, u. zwar schneidet die letztere Gerade

$$
\alpha x_{1}+x_{3}+\beta x_{2}=0
$$


die Gerade $x_{1}=0$ in $H$. Der reelle Theil der ersten Gleichung stellt also die Gerade $C H^{\prime}$ dar.

Der reelle und imaginäre Theil der zweiten Gleichung stellen conjugierte Gerade $h, h^{\prime}$ der Involution $H \Re$ dar; diese Involution erhielten wir aus $D \mathscr{A}$, indem wir den Schnittpunkt von $D \mathfrak{A}$ und $B C$ mit $H$ verbanden; verbinden wir statt dessen mit $H$ den Schnittpunkt von $B C$ und der Geraden, deren Gleichung durch den reellen oder imaginären Theil von

$$
\left(\alpha \eta_{1}+\eta_{3}-\mu i \eta_{2}\right)\left[\left(\eta_{3}+\mu i \eta_{2}\right) x_{1}-\eta_{1}\left(x_{3}+\mu i x_{2}\right)\right]=0
$$

gegeben ist, so erhaiten wir die Geraden $h, h^{\prime}$. Der imaginäre Theil der zuletzt angeschriebenen Gleichung ist:

$$
\begin{gathered}
\eta_{2}\left(\eta_{1} x_{3}-\eta_{3} x_{1}\right)-\left(\alpha \eta_{1}+\eta_{3}\right)\left(\eta_{2} x_{1}-\eta_{1} x_{2}\right)=0 \\
\left(\alpha \eta_{1}+\eta_{3}\right) x_{2}-\eta_{2}\left(\alpha x_{1}+x_{3}\right)=0
\end{gathered}
$$

Er stellt die Gerade $C D E$ dar, daher stellt der reelle Theil jener Gleichung die Gerade $D E^{\prime}$ dar. Ist $C^{\prime}$ der Schnittpunkt von $B C$ mit $D E^{\prime}$, so ist $H C^{\prime}$ die Gerade $h$, womit sich für die Gerade $D X$ die oben angegebene Construction ergibt.

Eine andere Construction können wir aus der Gleichung von $D X$ herleiten, wenn wir den Schnittpunkt $K$ dieser Geraden mit $x_{1}=0$ suchen. Durch $K$ geht nämlich auch die Gerade, deren Gleichung mit dem reellen Theil der folgenden ïbereinstimmt:

$$
\begin{gathered}
\eta_{1} \eta_{2}(\mu i-\beta)\left(\alpha x_{1}+x_{3}-\mu i x_{2}\right)+\left(\alpha \eta_{1}+\eta_{3}-\mu i \eta_{2}\right) \eta_{1}\left(x_{3}+\beta x_{2}\right) \\
\equiv \eta_{1}(\mu i-\beta)\left[\eta_{2}\left(\alpha x_{1}+x_{3}-\mu i x_{2}\right)-\left(\alpha \eta_{1}+\eta_{3}-\mu i \eta_{2}\right) x_{2}\right]+ \\
+\eta_{1}\left(\alpha \eta_{1}+\eta_{3}-\mu i \eta_{2}\right)\left(x_{3}+\mu i x_{2}\right)=0 .
\end{gathered}
$$

Aus der ersten Darstellung folgt, dass die gesuchte Gerade durch den Schnittpunkt $M$ von $C H^{\prime}$ mit $B H$ geht, aus der zweiten, dass sie durch den Schnittpunkt $N$ von $C D$ mit $B E^{\prime}$ geht. Daraus ergibt sich folgende Construction: Haben $H, H^{\prime}, E, E^{\prime}$ dieselbe Bedeutung wie in derersten Construction, so suche man die Schnittpunkte:

$$
\left.\left.\left.\begin{array}{ll}
C H^{\prime} \\
B I I
\end{array}\right\} M \quad \begin{array}{ll}
C D & B E^{\prime}
\end{array}\right\} N \quad \begin{array}{l}
M N \\
\mathfrak{Q} \mathfrak{F}^{*}
\end{array}\right\} K
$$

$D K$ schneidet $g$ in $X$.

\section{Anfgabe.}

Von einem Kegelschnitte sind zwei Paare complex conjugierter Punkte $\mathfrak{A P}^{*}$ und $\mathfrak{B}^{*} \mathfrak{B}^{*}$ und ein reeller Punkt $C$ gegeben. 
Es soll der zweite Curvenpunkt auf einer beliebigen durch $C$ gehenden reellen Geraden $g$ construiert werden.

Es sei $B_{3}$ der Schnittpunkt der reellen Träger der Punkte $\mathfrak{A}, \mathfrak{B} ; B_{2}$ und $B_{1}$ seien demselben in den Involutionen $\mathfrak{A}$, bezw. $\mathfrak{B}$ conjugiert. $B_{1}, B_{2}, B_{3}$ seien die Eckpunkte des Coordinatendreiecks. Dann ist die analytische Darstellung der gegebenen Elemente :

$$
\begin{array}{lll}
\mathfrak{A} & x_{1}=0 & x_{3}+\mu i x_{2}=0 \\
\mathfrak{H}^{*} & x_{1}=0 & x_{3}-\mu i x_{2}=0 \\
\mathfrak{B}^{*} & x_{2}=0 & x_{3}+\nu i x_{1}=0 \\
\mathfrak{B}^{*} & x_{2}=0 & x_{3}-\nu i x_{1}=0 \\
C & \varrho x_{1}=\eta_{1}, \quad \varrho x_{2}=\eta_{2}, \quad \varrho x_{3}=\eta_{3} \quad \eta_{1} \geq 0 \quad \eta_{2} \geq 0 \\
C g & \left(\alpha \eta_{1}+\beta \eta_{2}\right) & x_{3}-\eta_{8}\left(\alpha x_{1}+\beta x_{2}\right)=0
\end{array}
$$

Nach dem auf das Sechseck $\mathfrak{A} \mathfrak{A}^{*} \mathfrak{B} \mathfrak{B}^{*} C X$ angewandten Pas cal'schen Satze liegen auf einer Geraden die Punkte $\mathfrak{h}, \mathfrak{F}, \mathfrak{A}$ :

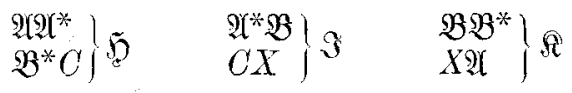

$$
\left.\begin{array}{ll}
\mathfrak{H} \mathfrak{H}^{*} & x_{1}=0 \\
C \mathfrak{B}^{*} & \left(\eta_{3}-\nu i \eta_{1}\right) x_{2}-\eta_{2}\left(x_{3}-\nu i x_{1}\right)=0 \\
\mathfrak{A}^{*} \mathfrak{B} & x_{3}-\mu i x_{2}+\nu i x_{1}=0 \\
O g & \left(\alpha \eta_{1}+\beta \eta_{2}\right) x_{\mathfrak{3}}-\eta_{3}\left(\alpha x_{1}+\beta x_{2}\right)=0
\end{array}\right\} \mathfrak{S}
$$

$\mathfrak{5} 3 a\left[\left(\alpha \eta_{1}+\beta \eta_{2}\right) x_{3}-\eta_{3}\left(\alpha x_{1}+\beta x_{2}\right)\right]+b\left(x_{3}-\mu i x_{2}+v i x_{1}\right)$

$$
=c\left[\left(\eta_{3}-v i \eta_{1}\right) x_{2}-\eta_{2}\left(x_{3}-v i x_{1}\right)\right]+d x_{1}=0
$$

Die Constanten $a, b$ sind so zu bestimmen, dass die Gleichung durch $x_{1}=0, x_{2}=\eta_{2}, x_{3}=\eta_{3}-\nu i \eta_{1}$ befriedigt wird.

$$
a\left[\left(\alpha \eta_{1}+\beta \eta_{2}\right)\left(\eta_{3}-\nu i \eta_{1}\right)-\eta_{2} \eta_{3} \beta\right]=-b\left[\eta_{3}-\mu i \eta_{2}-\nu i \eta_{1}\right] .
$$

Setzt man in die beiden Gleichungsformen für $\mathfrak{\mathfrak { s }}\} x_{1}=0$, $x_{3}=\mu i x_{2}$ ein, so erhält man:

$$
a\left[\left(\alpha \eta_{1}+\beta \eta_{2}\right) \mu i-\beta \eta_{3}\right]=c\left[\eta_{3}-\nu i \eta_{1}-\mu i \eta_{2}\right] .
$$

Die Constante $d$ kann man bestimmen, indem man $x_{r}=\eta_{r}$ einsetzt; wir brauchen $d$ jedoch nicht.

Wir haben also für $\mathfrak{5} \Im$ die Gleichung:

$$
\begin{gathered}
\left(\eta_{3}-\mu i \eta_{2}-\nu i \eta_{1}\right)\left[\left(\alpha \eta_{1}+\beta \eta_{2}\right) x_{3}-\eta_{3}\left(\alpha x_{1}+\beta x_{2}\right)\right]-\left[\alpha \eta_{1} \eta_{3}-\right. \\
\left.-\nu \eta_{1} i\left(\alpha \eta_{1}+\beta \eta_{2}\right)\right]\left(x_{3}-\mu i x_{2}+\nu i x_{1}\right) \\
\equiv\left[\beta \eta_{3}-\mu i\left(\alpha \eta_{1}+\beta \eta_{2}\right)\right] \cdot\left[\eta_{2}\left(x_{3}-\nu i x_{1}\right)-\left(\eta_{3}-\nu i \eta_{1}\right) x_{2}\right]+d x_{1}=0 .
\end{gathered}
$$


Der Schnittpunkt dieser Geraden mit $x_{2}=0$ ist der Punkt $\Re$.

$$
\begin{aligned}
& \mathscr{A} \mathfrak{A}\left\{\left[\beta \eta_{3}-\mu i\left(\alpha \eta_{1}+\beta \eta_{2}\right)\right] .\left[\eta_{2}\left(x_{3}-\nu i x_{1}\right)-\left(\eta_{3}-\nu i \eta_{1}\right) x_{2}\right]+d x_{1}\right\}+ \\
&+\left[\beta \eta_{3}-\mu i\left(\alpha \eta_{1}+\beta \eta_{2}\right)\right]\left[\mu i \eta_{2}+\eta_{3}-\nu i \eta_{1}\right] x_{3} \\
& \equiv \\
& \equiv {\left[\beta \eta_{3}-\mu i\left(\alpha \eta_{1}+\beta \eta_{2}\right)\right] \eta_{2}\left(x_{3}+\mu i x_{2}\right)+e x_{1}=0 . }
\end{aligned}
$$

Der Coefficient von $\mu i \eta_{2}$ in dieser Gleichung ist, wie aus der letzten Form derselben folgt:

$$
\beta \eta_{3} x_{2}-\left(\alpha \eta_{1}+\beta \eta_{2}\right) x_{3}+\varepsilon x_{1} .
$$

Die Constante $\varepsilon$ ist der Coefficient von $\mu i \eta_{2} x_{1}$ in der Gleichung von $\mathfrak{Y} \mathfrak{Y}$ :

$$
\begin{aligned}
\mu i \eta_{2} \varepsilon & =\alpha \eta_{3}\left(\mu i \eta_{2}+\nu i \eta_{1}\right)-\nu i \alpha \eta_{1} \eta_{3}=\alpha \eta_{3} \eta_{3} \mu i \\
\varepsilon & =\alpha \eta_{3}
\end{aligned}
$$

Der imaginäre Theil der Gleichung von 2 (S ist also:

$$
\eta_{3}\left(\alpha x_{1}+\beta x_{2}\right)-\left(\alpha \eta_{1}+\beta \eta_{2}\right) x_{3}=0 .
$$

Es ist die Gleichung von $C g X$. Der reelle Punkt der Geraden $\mathfrak{H}_{\Omega}$ liegt also auf $C g$ : es ist der gesuchte Punkt $X$. Durch ihn gehen alle Strahlen der Involution $2 \Re$, also auch die Gerade $h$, deren Gleichung mit dem reellen Theile der Gleichung für $\mathcal{A} \Re$ übereinstimmt. Schneidet $g$ die Gerade $x_{1}=0$ in $G_{1}$ und ist $G_{1}^{\prime}$ in der Involution $\mathfrak{A} \mathrm{zu} G_{1}$ conjugiert, so geht $h$ durch $G_{1}^{\prime}$. Ist $G_{2}$ der Schnittpunkt von $g$ mit $x_{2}=0$, $G_{2}^{\prime}$ der zu $G_{2}$ in der Involution $\mathfrak{B}, G_{2}^{\prime \prime}$ der zu $G_{2}$ in der Involution $\Re$ conjugierte Punkt, so geht $h$ dureh $G_{2}^{\prime \prime}$. Durch $G_{\text {.2 }}^{\prime \prime}$ geht, wie aus der analytischen Darstellung von $\mathfrak{\Omega}$ ersichtlich ist, auch die Gerade, deren Gleichung mit dem reellen Theile der folgenden übereinstimmt:

$$
\begin{gathered}
\eta_{3}\left[\left(\alpha \eta_{1}+\beta \eta_{2}\right) x_{3}-\eta_{3}\left(\alpha x_{1}+\beta x_{2}\right)\right]- \\
-\left[\alpha \eta_{1} \eta_{3}-\nu \eta_{1} i\left(\alpha \eta_{1}+\beta \eta_{2}\right)\right]\left(x_{3}+\nu i x_{1}\right)=0 .
\end{gathered}
$$

Die letztgenannte Gerade geht durch den Schnittpunkt $K$ von $C g$ mit $B_{2} G_{2}^{\prime}$ und den. Schnittpunkt $L$ von $x_{1}=0$ mit $\eta_{2} x_{3}-\eta_{3} x_{2}=0$, das ist mit $C B_{1}$. Der erste Theil dieser Behauptung folgt daraus, dass der imaginäre Theil der Gleichung:

$$
\left[\alpha \eta_{3}-\nu i\left(\alpha \eta_{1}+\beta \eta_{2}\right)\right]\left(x_{s}+\nu i x_{1}\right)=0
$$

die Gerade $B_{2} G_{2}$ darstellt.

Wir erhielten somit folgende Construction. "Sind $G_{1}$ und $G_{2}$ die Schnittpunktevon $C g$ mit $\mathfrak{H}^{*}$, beziehungsweise $\mathfrak{B B}^{*}$, ist $B_{3}$ der Schnittpunkt von $\mathfrak{A H}^{*}$ mit $\mathfrak{B}_{\mathfrak{B}^{*}}$, 
ist ferner dercomplexe Punkt $\mathfrak{A}$ durch die Punktepaare $B_{3} B_{2}, G_{1} G_{1}^{\prime}$, der Punkt $\mathfrak{B}$ durch die Punktepaare $B_{3} B_{1}, G_{2} G_{2}^{\prime}$ definiert: so suchemandie Schnittpunkte:

$$
\left.\begin{array}{lll}
C g & B_{2} B_{3} \\
B_{2} G_{2}^{\prime}
\end{array} \mid L \quad \begin{array}{ll}
B_{1} B_{3} \\
C B_{1} \mid
\end{array}\right\} G_{2}^{\prime \prime}
$$

$G_{2}^{\prime \prime} G_{3}^{\prime}$ schneidet $g$ im gesuchten Punkte $x$."

Eine andere Construction erhalten wir, wenn wir in den Involutionen $\mathfrak{A}, \mathfrak{A}$ je den zu $B_{3}$ conjugierten Punkt suchen; der erste ist $B_{2}$, der zweite sei $B_{3}^{\prime}$. Multipliciert man die Gleichung. von $\mathscr{X} \Re$ mit $\beta \eta_{3}+\mu i\left(\alpha \eta_{1}+\beta \eta_{2}\right)$, so stellt der imaginäre Theil der erhaltenen Gleichung eine durch $B_{3}$ gehende Gerade dar. Der reelle Theil stellt also die gerade $B_{2} B_{3}$ dar. Um den Punkt $B_{3}^{\prime}$ zu exhalten, haben wir die Gleichung von $\mathfrak{5} \Im$ mit demselben Coefficienten $\beta \eta_{3}+\mu i\left(\alpha \eta_{1}+\beta \eta_{2}\right)$ zu multiplicieren und den reellen Theil der so erhaltenen Gleichung zu deuten. Diese Gleichung stellt sich uns in zwei Formen dar:

$$
\begin{gathered}
f \cdot\left[\left(\alpha \eta_{1}+\beta \eta_{i}\right) x_{3}-\eta_{3}\left(\alpha x_{1}+\beta x_{2}\right)\right]- \\
-\left[\alpha \eta_{1} \eta_{3}-v \eta_{1} i\left(\alpha \eta_{1}+\beta \eta_{2}\right)\right]\left[\beta \eta_{3}+\mu i\left(\alpha \eta_{1}+\beta \eta_{2}\right)\right] . \\
\cdot\left(x_{3}-\mu i x_{2}+v i x_{1}\right)=0 . \\
\text { u. }\left[\left(\beta \eta_{3}\right)^{2}+\mu^{2}\left(\alpha \eta_{1}+\beta \eta_{2}\right)^{2}\right]\left[\eta_{2}\left(x_{3}-v i x_{1}\right)-\left(\eta_{3}-v i \eta_{1}\right) x_{2}\right]+b^{\prime} x_{1}=0 .
\end{gathered}
$$

Ans (II) ist ersichtlich, dass der gesuchte Strahl der Involution $\mathfrak{h} \mathfrak{F}$ durch den Schnittpunkt $L$ von $C B_{1}$ mit $B_{2} B_{3}$ geht. Um die Form (I) zu deuten, beachten wir, dass der imaginäre, beziehungsweise reelle Theil von

$$
\left[\alpha \eta_{1} \eta_{3}-\nu \eta_{1} i\left(\alpha \eta_{1}+\beta \eta_{2}\right)\right]\left(x_{3}+\nu i x_{1}\right)=0
$$

mit $x_{2}=0$ die Coordinaten von $G_{2}$, beziehungsweise $G_{2}^{\prime}$ liefert, der imaginäre, beziehungsweise reelle Theil von

$$
\left[\beta \eta_{3}+\mu i\left(\alpha \eta_{1}+\beta \eta_{2}\right)\right]\left(x_{3}-\mu i x_{2}\right)=0
$$

mit $x_{1}=0$ die Coordinaten von $G_{1}$, beziehungsweise $G_{1}^{\prime}$. Der reelle. Theil des Ausdruckes

$$
\left[\alpha \eta_{3}-v i\left(\alpha \eta_{1}+\beta \eta_{2}\right)\right]\left[\beta \eta_{3}+\mu i\left(\alpha \eta_{1}+\beta \eta_{2}\right)\right]\left(x_{3}-\mu i x_{2}+\nu i x_{1}\right)
$$
ist:

$$
\begin{aligned}
& \alpha \beta \eta_{3}^{2} x_{3}+\alpha \eta_{3}\left(\alpha \eta_{1}+\beta \eta_{2}\right) \mu^{2} x_{2}+\beta \eta_{3}\left(\alpha \eta_{1}+\beta \eta_{2}\right) \nu^{2} x_{1}+ \\
& \quad+\mu \nu\left[\left(\alpha \eta_{1}+\beta \eta_{2}\right) x_{3}-\eta_{3}\left(\alpha x_{1}+\beta x_{2}\right)\right]\left(\alpha \eta_{1}+\beta \eta_{2}\right) .
\end{aligned}
$$

Wir können somit den reellen Theil von (I) auch in folgender Form schreiben: 


$$
\begin{gathered}
f^{\prime} \cdot\left[\left(\alpha \eta_{1}+\beta \eta_{2}\right) x_{3}-\eta_{3}\left(\alpha x_{1}+\beta x_{2}\right)\right]- \\
-\eta_{3}\left[\alpha \beta \eta_{3} x_{3}+\alpha\left(\alpha \eta_{1}+\beta \eta_{2}\right) \mu^{2} x_{2}+\beta\left(\alpha \eta_{1}+\beta \eta_{2}\right) \nu^{2} x_{1}\right]=0 .
\end{gathered}
$$

Der zweite Theil gleich Null gesetzt ist die Gleichung von $G_{1}^{\prime} G_{2}^{\prime}$. Die gesuchte Gerade von $\mathfrak{g} \Im$ geht also durch den Schnittpunzt $G$ von $g$ mit $G_{1}^{\prime} G_{2}^{\prime}$.

Wir erhalten also folgende Construction von $X$. "H a be $\mathrm{n}$ $B_{1} B_{2} B, G_{1}, G_{1}^{\prime}, G_{2}, G_{2}^{\prime}$ die oben angegebene Bedeutung, so suche man die Schnittpunkte:

$$
\left.\left.\left.\begin{array}{l}
C B_{1} \\
B_{2} B_{3}
\end{array}\right\} L \quad \begin{array}{l}
C g \\
G_{1} G_{2}
\end{array}\right\} G \quad \begin{array}{l}
G L \\
B_{1} B_{3}
\end{array}\right\} B_{3} .
$$

Die Gerade $B_{2} B_{3}^{\prime}$ schneidet $g$ im gesuchten Punkte $X$. Diese Construction ist die von $\mathrm{Stolz}$ angegebene. 